\title{
An effective potential for one-dimensional matter-wave solitons in an axially inhomogeneous trap
}

\author{
Sergio De Nicola ${ }^{1}$, Boris A. Malomed ${ }^{2}$, Renato Fedele ${ }^{3}$ \\ ${ }^{1}$ Istituto di Cibernetica "E Caianiello " del CNR, Comprensorio "A. Olivetti", \\ Via Campi Flegrei, 34, I-80078 Pozzuoli (Na), Italy \\ ${ }^{2}$ Department of Interdisciplinary Studies, School of Electrical Engineering, \\ Faculty of Engineering, Tel Aviv University, Tel Aviv 69978, Israel and \\ ${ }^{3}$ Dipartimento di Scienze Fisiche, Universitá Federico II and INFN Sezione di Napoli, \\ Complesso Universitario di M.S. Angelo, via Cintia, I-80126, Napoli, Italy
}

\begin{abstract}
We demonstrate that a tight transverse trap with the local frequency, $\omega_{\perp}$, gradually varying in the longitudinal direction $(x)$ induces an effective potential for one-dimensional solitons in a selfattractive Bose-Einstein condensate. An analytical approximation for this potential is derived by means of a variational method. In the lowest approximation, the potential is $N(S+1) \omega_{\perp}(x)$, with $N$ the soliton's norm (number of atoms), and $S$ its intrinsic vorticity (if any). The results can be used to devise nonuniform traps helping to control the longitudinal dynamics of the solitons. Numerical verification of the analytical predictions will be presented elsewhere.
\end{abstract}

To be published in Physics Letters A

\section{INTRODUCTION}

A Bose-Einstein condensate (BEC) with attractive interactions between atoms (negative scattering length) may be stable in an external trap if the number of atoms in the condensate is below a collapse threshold [1]. In this case, the BEC in a nearly one-dimensional (1D) "cigar-shaped" trap, which features tight confinement in the transverse plane and a weak potential along the longitudinal axis, can form stable matter-wave packets in the form of bright solitons. A single soliton [2] and multi-soliton complexes [3] were created in the ${ }^{7} \mathrm{Li}$ condensate loaded into a strongly elongated optical trap. More recently, solitons whose shape is nearly three-dimensional (3D), were observed in a post-collapse state in a condensate of ${ }^{85} \mathrm{Rb}$ atoms [4] (as well as in ${ }^{7} \mathrm{Li}$, in this experiment the sign of the interatomic interactions was switched into attractive by means of the Feshbach resonance).

A fundamental equation which provides for a very accurate description of the dynamics of a rarefied quantum gas of Boson atoms in the mean-field approximation is the 3D Gross-Pitaevskii (GP) equation [1]. In a normalized form, the equation is

$$
i \frac{\partial \psi}{\partial t}=-\frac{1}{2}\left(\nabla_{\perp}^{2}+\frac{\partial^{2}}{\partial x^{2}}\right) \psi+\left[\frac{1}{2} \omega_{\perp}^{2}\left(y^{2}+z^{2}\right)+U(x)\right] \psi+g|\psi|^{2} \psi,
$$

where $\psi$ is the single-atom wave function, $x$ and $y, z$ are the longitudinal (axial) and transverse coordinates (the transverse Laplacian $\nabla_{\perp}^{2}$ acts on $y$ and $\left.z\right), \omega_{\perp}$ is the frequency accounting for the tight transverse confinement, $U(x)$ a loose axial potential, and $g$ a scaled nonlinearity constant (in the case of self-attraction, $g$ is negative).

In the experiment, the transverse trapping potential may be axially nonuniform, which corresponds to $\omega_{\perp}=\omega_{\perp}(x)$ in Eq. (11). It may also depend on time, hence $\omega_{\perp}=\omega_{\perp}(x, t)$, in the most general case. Actually, the axial nonuniformity is an unavoidable feature of any experimental setup, and, on the other hand, specially designed nonuniformity may be used as an additional tool for the control of dynamics of trapped solitons. The objective of this Letter is to derive an effective longitudinal potential induced (in addition to the explicitly present potential, $U(x)$ in Eq. (1D) by the $x$-dependence of $\omega_{\perp}$, and the corresponding equation of motion for axial solitons.

If the transverse potential is much stronger than the longitudinal one, it is natural to reduce the full 3D GP equation, (11), to an effective 1D equation. Different approaches were proposed to achieve this purpose [5]-[9] under different conditions. In most cases, the reduction is based on assuming a factorized ansatz for the wave function [5, 6],

$$
\psi(x, y, z, t)=\exp \left(-\frac{r^{2}}{2 \sigma^{2}(x, t)}\right) \frac{f(x, t)}{\sqrt{\pi} \sigma(x, t)},
$$

where $r^{2} \equiv y^{2}+z^{2}$, the transverse width $\sigma$ and local amplitude $f$ being slowly varying functions of $x$ and $t$. Using the Lagrangian representation of Eq. (1), effective 1D equations for $\sigma$ and $f$ can be derived as variational equations. First, $\sigma$ is eliminated in favor of $f$,

$$
\sigma^{2}=\omega_{\perp}^{-1} \sqrt{1+\left(g / 2 \pi \omega_{\perp}\right)|f|^{2}}
$$


and then a closed-form 1D GP equation with nonpolynomial nonlinearity is derived for $f$ [6]:

$$
i \frac{\partial f}{\partial t}=-\frac{1}{2} \frac{\partial^{2} f}{\partial x^{2}}+\left[\omega_{\perp} \frac{1+\left(3 g / 4 \pi \omega_{\perp}\right)|f|^{2}}{\sqrt{1+\left(g / 2 \pi \omega_{\perp}\right)|f|^{2}}}+U(x)\right] f .
$$

Equation (4) with $g<0$ admits stable solitary-wave solutions, which were analyzed in Ref. [6] too.

In the case of the weak nonlinearity, $\left(g / 2 \pi \omega_{\perp}\right)|f|^{2} \ll 1$, the nonlinear term in Eq. (4) may be expanded in powers of $|f|^{2}$, which leads to the cubic GP equation with an additional quintic term that corresponds to higher-order self-attraction,

$$
i \frac{\partial f}{\partial t}=-\frac{1}{2} \frac{\partial^{2} f}{\partial x^{2}}+\omega_{\perp}\left(\frac{g}{2 \pi \omega_{\perp}}|f|^{2}-\frac{3 g^{2}}{8 \pi^{2} \omega_{\perp}^{2}}|f|^{4}\right) f+\left[\omega_{\perp}+U(x)\right] f .
$$

In a more direct form, a 1D equation with the cubic-quintic (CQ) nonlinearity of this type was derived and employed in Refs. 5, 9]. A formally similar equation of the CQ type (but with a self-defocusing quintic term) was considered earlier in works aiming to take into regard three-body collisions in BEC [10]). Despite the possibility of collapse induced by the self-focusing quintic term in the 1D setting, the CQ equation has a family of exact soliton solutions, which are stable against small perturbations [11] (these new solutions were obtained as an analytical continuation of well-known soliton solutions [12] to the CQ equation with the self-defocusing quintic term).

The approach based on the factorized ansatz (2) was generalized in Ref. [8] to describe configurations with intrinsic vorticity, with the ansatz replaced by

$$
\psi(x, y, z, t)=r^{S} \exp \left(-\frac{r^{2}}{2 \sigma^{2}(x, t)}+i S \theta\right) f_{S}(x, t)
$$

where $\theta$ is the angular coordinate in the $(y, z)$ plane, and $S=1,2, \ldots$ is the integer vorticity (which naturally carries with itself the pre-exponential factor $r^{S}$ ). Actually, $S$ plays the role of the "spin" of effectively 1D solitons generated by the ansatz. This way, an equation for $f_{S}(x, t)$ similar to Eq. (4) can be derived.

The Letter is organized as follows. In the next section, using the variational approximation [13], we develop a framework for the analysis of the soliton's dynamics in the model with the axially nonuniform trapping, $\omega_{\perp}=\omega_{\perp}(x)$ (the variational approach employs the Hamiltonian, rather than Lagrangian). In Section 3, we derive a final result, viz., an effective axial potential for the soliton induced by the $x$-dependence of $\omega_{\perp}$, and the corresponding equation of motion for the soliton. The analytical results are obtained under the natural assumption that a scale of the variation of $\omega_{\perp}(x)$ is much larger than the size of the soliton. The paper is concluded by Section 4 .

\section{VARIATIONAL ANALYSIS}

Aiming to derive an effective potential and equation of motion for solitons in the case of $\omega_{\perp}=\omega_{\perp}(x)$, we skip the derivation of an effective 1D equation, and instead adopt a 3D ansatz for the soliton which, in the general case, includes the intrinsic vorticity (cf. Eq. (6) ) :

$$
\psi_{\mathrm{sol}}=A r^{S} \exp \left(-\frac{r^{2}}{2 \sigma^{2}}+i S \theta\right) \operatorname{sech}\left(\frac{x-\xi}{W}\right) e^{i \phi},
$$

with amplitude $A$, longitudinal width $W$, central coordinate $\xi$ and phase $\phi$, in addition to the transverse width, $\sigma$, and spin, $S=0,1,2, \ldots$, that were defined above. The norm of this ansatz is

$$
N \equiv 2 \pi \int_{0}^{\infty} r d r \int_{-\infty}^{+\infty}\left|\psi_{\text {sol }}(r, x)\right|^{2}=2 \pi S ! A^{2} W \sigma^{2(S+1)}
$$

To derive an effective potential for the soliton as a function of the coordinate $\xi$, we use the Hamiltonian of threedimensional equation (11),

$$
H=\pi \int_{-\infty}^{+\infty} d x \int_{0}^{\infty} r d r\left[\left|\psi_{x}\right|^{2}+\left|\psi_{r}\right|^{2}+\left(\omega_{\perp}^{2}(x) r^{2}+2 U(x)\right)|\psi|^{2}+g|\psi|^{4}\right] .
$$


The substitution of ansatz (7) in $H$ and straightforward integrations yield

$$
\begin{aligned}
\frac{H}{\pi}= & (1+S) ! A^{2} W \sigma^{2 S}+\frac{S !}{3} \frac{A^{2}}{W} \sigma^{2(1+S)}+(1+S) ! \omega_{\perp}^{2}(\xi) A^{2} W \sigma^{2(2+S)} \\
& +2 S ! U(\xi) A^{2} W \sigma^{2(1+S)}+\frac{g}{3} \frac{(2 S) !}{2^{2 S}} A^{4} W \sigma^{2(1+2 S)} .
\end{aligned}
$$

This result was obtained under the condition that, as said above, $\omega_{\perp}(x)$ varies on a scale which is much longer than the soliton's width, $W$, the same being assumed about the axial potential, $U(x)$. For this reason, $\omega_{\perp}^{2}(x)$ and $U(x)$ in Hamiltonian (10) are taken at $x=\xi$.

The soliton's peak density, $A^{2}$, can be eliminated, in Eq. (10), in favor of the conserved norm, $N$, using Eq. (8). This leads to

$$
\begin{aligned}
H & =(1+S) \frac{N}{2}\left[\frac{1}{\sigma^{2}(\xi)}+\omega_{\perp}^{2}(\xi) \sigma^{2}(\xi)\right]+\frac{1}{6} \frac{N}{W^{2}}+N U(\xi)+\frac{C_{S}}{3 \pi} \frac{g N^{2}}{W \sigma^{2}}, \\
C_{S} & \equiv \frac{(2 S) !}{2^{2(1+S)}(S !)^{2}} .
\end{aligned}
$$

In this expression, the transverse and longitudinal widths of the soliton, $\sigma$ and $W$, are free parameters. The soliton chooses their values by minimizing the Hamiltonian, which leads to conditions

$$
\frac{\partial H}{\partial W}=\frac{\partial H}{\partial\left(\sigma^{2}\right)}=0
$$

The first condition takes a simple form, making it possible to eliminate the longitudinal width,

$$
W=-\frac{\pi}{C_{S}} \frac{\sigma^{2}}{g N}
$$

which, obviously, makes sense only for $g<0$. Indeed, bright solitons may only exist in the BEC with the attractive nonlinearity, corresponding to negative $g$ (unless a periodic optical-lattice potential is present, that can support gap solitons at $g>0$ [14, 15]). The substitution of $W$ from expression (14) in Eq. (11) yields

$$
H=(1+S) \frac{N}{2}\left[\frac{1}{\sigma^{2}(\xi)}+\omega_{\perp}^{2}(\xi) \sigma^{2}(\xi)\right]+N U(\xi)-\frac{C_{s}^{2}}{6 \pi^{2}} \frac{g^{2} N^{3}}{\sigma^{4}}
$$

Further, the second condition from Eq. (13) amounts to a cubic equation for $\sigma^{-2}$ :

$$
\frac{2 C_{s}^{2}}{3 \pi^{2}(1+S)} \frac{(g N)^{2}}{\left(\sigma^{2}\right)^{3}}-\frac{1}{\left(\sigma^{2}\right)^{2}}+\omega_{\perp}^{2}=0
$$

It is easy to see that Eq. (16) has physical (positive) solutions for $\sigma^{2}$ if the normalized number of atoms is smaller than a critical value,

$$
N^{2}<N_{\mathrm{cr}}^{2}\left(\omega_{\perp}\right) \equiv \frac{\pi^{2}(1+S)}{\sqrt{3} C_{s}^{2} g^{2} \omega_{\perp}} .
$$

The existence of largest $N$ beyond which stationary solutions do not exist reflects an obvious fact that the underlying axisymmetric 3D GP equation (11) gives rise to collapse if $N$ is too large [16]. Equations (17) and (12) predict increase of $N_{\mathrm{cr}}$ (for given $\omega_{\perp}$ ) in the lowest vortex state $(S=1)$, in comparison with its zero-vorticity counterpart, by a factor of $2 \sqrt{2}$, which is a known effect too, see Ref. [17] and references therein.

Alternatively, Eq. (17) demonstrates that, for given $N$, physical solutions exist if the transverse confinement is not too strong (otherwise, over-squeezing of the condensate will lead to the collapse):

$$
\omega_{\perp}<\left(\omega_{\perp}\right)_{\max } \equiv \frac{\pi^{2}(1+S)}{\sqrt{3}\left(C_{s} g N\right)^{2}} .
$$

If there is a region where $\omega_{\perp}(x)$ exceeds $\left(\omega_{\perp}\right)_{\max }$, a moving soliton crossing into this region will blow up due to the collapse. 
Further analysis of Eq. (16) demonstrates that there are two solutions for $\sigma^{2}$, one decreasing with $\omega_{\perp}$, and the other one increasing. The former behavior is natural (stronger squeeze leads to a smaller transverse size of the condensate), while the latter one is not. To all appearance, the latter solution is unstable. Therefore, we only take into regard the solutions with $\sigma\left(\omega_{\perp}\right)$ a decreasing function. In particular, it is easy to demonstrate that this solution yields, for given $N$, the transverse width in the range of

$$
\frac{C_{s}|g| N}{\pi \sqrt{1+S}} \equiv \sigma_{\min } \leq \sigma<\infty
$$

Here, $\sigma_{\min }$ corresponds to $\omega_{\perp}=\left(\omega_{\perp}\right)_{\max }$ in Eq. (18).

\section{THE EFFECTIVE POTENTIAL AND EQUATION OF MOTION FOR THE SOLITON}

If $\sigma^{2}$ can be found from Eq. (16), then $W$ must be taken as per Eq. (14), and both substituted in Hamiltonian (11). An explicit result can be obtained in the case of a relatively weak nonlinearity,

$$
\omega_{\perp}(g N)^{2} \ll \frac{3 \pi^{2}(1+S)}{C_{S}^{2}},
$$

when a relevant solution to Eq. (16), calculated to first two orders of the perturbative expansion, is

$$
\sigma^{2} \approx \frac{1}{\omega_{\perp}}-\frac{\left(C_{S} g N\right)^{2}}{3 \pi^{2}(1+S)}
$$

(note that the first term does not depend on $S$ ). Then, Eq. (15) yields the effective potential

$$
U_{\mathrm{eff}}(\xi) \approx N\left[(1+S) \omega_{\perp}(\xi)+U(\xi)-\frac{C_{s}^{2}}{6 \pi^{2}}(g N)^{2} \omega_{\perp}^{2}(\xi)\right] .
$$

For $S=0$, the appearance of combination $\omega_{\perp}(\xi)+U(\xi)$ in potential (21) might be expected from the form of the last term in Eq. (5). Equation (21) explicitly shows how the axially nonuniform transverse trapping induces the axial potential (which depends on the soliton's "spin"). This result may be realized as transformation of a part of the energy of the transverse motion into the axial potential when the confinement frequency gradually varies along $x$.

It is well known that, in the notation adopted here, an effective mass of the soliton, if it is treated as a quasiparticle, is $M_{\text {eff }}=2 N$ 13] (this relation does not depend on $S$ ). Thus, the equation of motion for the soliton in effective potential (21), $M_{\text {eff }}\left(d^{2} \xi / d t^{2}\right)=-\partial U_{\text {eff }} / \partial \xi$, takes the form

$$
\frac{d^{2} \xi}{d t^{2}}=-\frac{1}{2}\left[(1+S) \frac{d \omega_{\perp}}{d \xi}+\frac{d U}{d \xi}-\frac{C_{s}^{2}}{6 \pi^{2}}(g N)^{2} \frac{d\left(\omega_{\perp}^{2}\right)}{d \xi}\right] .
$$

This equation clearly shows that, in the absence of the explicit potential, $d U / d \xi=0$, stable and unstable equilibria for the soliton are positions where the local trapping frequency attains its minimum and maximum, respectively. As said above, the trapping frequency may also be time-dependent (in addition to being $x$-dependent), $\omega_{\perp}=\omega_{\perp}(\xi, t)$, then the right-hand side of Eq. (22) will explicitly depend on $t$.

In the most general case, without assuming condition (19) to hold, Eq. (16) can be solved for $\sigma^{2}$ numerically, and the effective potential can also be found in a numerical form. To illustrate this possibility, in Fig. 1 we display the dependence of $\sigma^{2}$ vs. $N$, as obtained from the numerical solution of Eq. (16) at several fixed values of $\omega_{\perp}$, with $S=0$. As seen from the figure, the transverse width gradually decreases with the increase of $N$, as predicted by Eq. (20). The latter effect is a natural manifestation of the self-squeezing of the condensate with attraction between atoms.

Results obtained by the substitution of the numerically found $\sigma^{2}$ in Hamiltonian (15) (with $S=0$ ), i.e., the effective potential, are displayed in Fig. 2. It is seen from this figure that both the linear dependence of the potential on $N$ for small $N$ and deviations from the linear dependence at larger $N$ follow the analytical prediction (21).

\section{CONCLUSIONS}

In this work, we have demonstrated that a tight transverse trap, whose strength gradually varies in the longitudinal (axial) direction, induces an effective longitudinal potential for the soliton in the self-attractive BEC. An analytical 


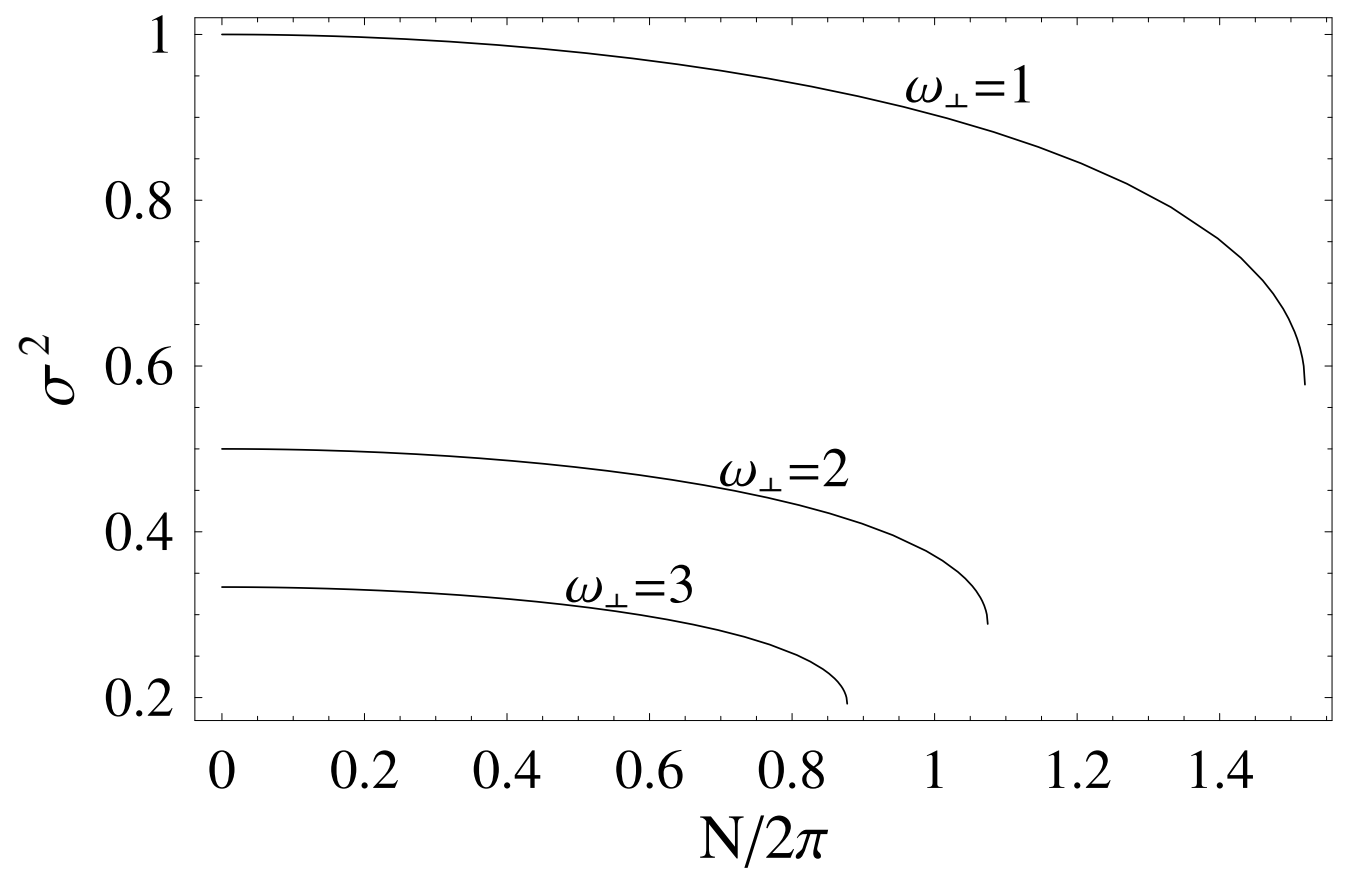

FIG. 1: The squared transverse size of the condensate, $\sigma^{2}$, as a function of norm $N$, at several fixed values of the transverse trapping frequency, $\omega_{\perp}$. The curves are obtained from numerical solution of Eq. 16 with $S=0$ and $g^{2}=1$, and terminate at $N=N_{\mathrm{cr}}\left(\omega_{\perp}\right)$, as per Eq. [17).

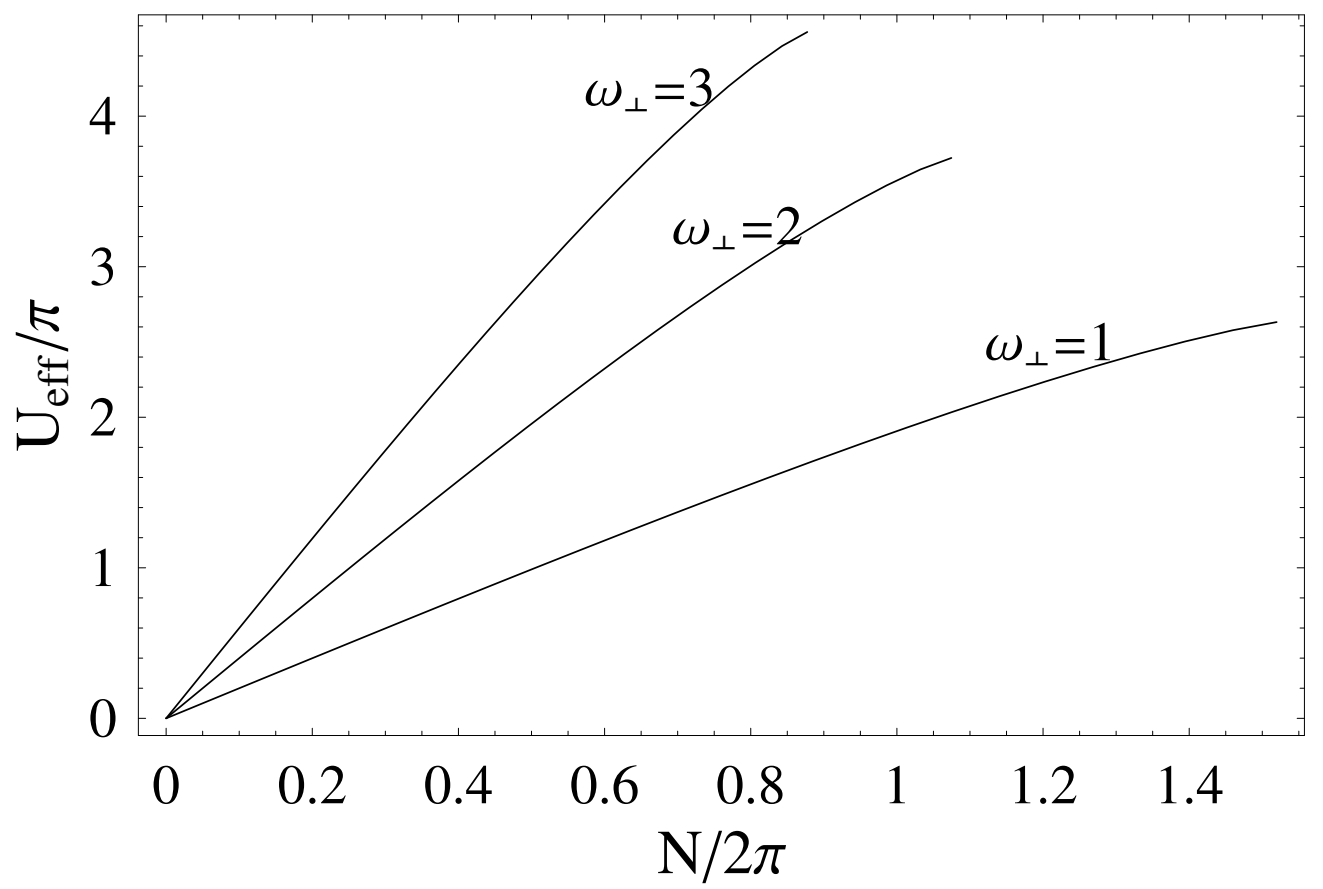

FIG. 2: Dependences of the effective potential (15) with $S=0, U=0$ and $g^{2}=1$ on the soliton's norm $N$ at several fixed values of $\omega_{\perp}$, as obtained by the substitution of numerical data for $\sigma^{2}$, which were used to generate Fig. 1 . The plots terminate at $N=N_{\mathrm{cr}}\left(\omega_{\perp}\right)$, the same way as in Fig. 1 . 
approximation for this potential, based on the variational method, was developed, under the assumption that the longitudinal size of the soliton is essentially smaller than the scale of the trap's nonuniformity. A fully explicit result was obtained for the case of weak nonlinearity. The potential depends on the soliton's intrinsic vorticity (if any), and its norm (the number of atoms trapped in the soliton). The analytical results reported in this Letter can be used to analyze inevitable effects of the nonuniformity of the transverse trapping in experimental situations, as well as to devise axially nonuniform traps with the purpose to control the longitudinal dynamics of the solitons. Systematic numerical simulations of the full 3D model, aimed at direct verification of the analytical predictions, demand special effort and will be reported elsewhere.

It is relevant to mention that a similar problem can also be considered in the model with repulsion $(g>0$ in Eq. (11) ) and a periodic term if the longitudinal potential generated by an optical lattice, $U_{\mathrm{OL}}(x)=\epsilon \cos (k x)$. In that case, the system can readily support bright solitons of the gap type [14, 15]. However, the effective dynamical mass of mobile gap solitons in negative [15]; for this reason, one may expect that, on the contrary to the ordinary solitons considered in this Letter, gap solitons will find their stable and unstable equilibria at points where the transversetrapping frequency attains, respectively, its maximum and minimum. These predictions are subject to verification in numerical simulations.

B.A.M. appreciates hospitality of the CNR Institute of Cybernetics "E Caianiello" (Pozzuoli, Italy) and Department of Physical Sciences at Universitá Federico II (Naples, Italy). The work of this author was supported, in a part, by the Israel Science Foundation, through the Center-of-Excellence grant No. 8006/03 (B.A.M.).

[1] C. J. Pethik, H. Smith, Bose-Einstein Condensation in Dilute Gases, Cambridge University Press, Cambridge, 2002.

[2] L. Khaykovich, F. Schreck, G. Ferrari, T. Bourdel, J. Cubizolles, L. D. Carr, Y. Castin, C. Salomon, Science 256 (2002) 1290 (2002).

[3] K. E. Strecker, G. B. Partridge, A. G. Truscott, R. G. Hulet, Nature 417 (2002) 150.

[4] S. L. Cornish, S. T. Thompson, C. E. Wieman, Phys. Rev. Lett. 96 (2006) 170401.

[5] A. E. Muryshev, G. V. Shlyapnikov, W. Ertmer, K. Sengstock, and M. Lewenstein, Phys. Rev. Lett. 89 (2002) 110401 (2002).

[6] L. Salasnich, A. Parola, and L. Reatto, Phys. Rev. A 65, 043614 (2002).

[7] Y. B. Band, I. Towers, and B. A. Malomed, Phys. Rev. A 67, 023602 (2003).

[8] L. Salasnich, cond-mat/0309071 (2003); Laser Physics 14 (2004) 291.

[9] S. Sinha, A. Y. Cherny, D. Kovrizhin, and J. Brand, Phys. Rev. Lett. 96 (2006) 030406.

[10] Yu. Kagan, A. E. Muryshev, and G. V. Shlyapnikov, Phys. Rev. Lett. 81 (1998) 933; F. Kh. Abdullaev, A. Gammal, L. Tomio, and T. Frederico, Phys. Rev. A 63 (2001) 043604.

[11] L. Khaykovich and B. A. Malomed, Deviation from one-dimensionality in stationary properties and collisional dynamics of matter-wave solitons, Phys. Rev. A, in press (2006).

[12] Kh. I. Pushkarov, D. I. Pushkarov, and I. V. Tomov, Opt. Quant. Electr. 11 (1979) 471; S. Cowan, R. H. Enns, S. S. Rangnekar, S. S. Sanghera, Can. J. Phys. 64 (1986) 311.

[13] B. A. Malomed, in: Progress in Optics 43, 71 (E. Wolf, editor: North Holland, Amsterdam, 2002).

[14] B. Eiermann, Th. Anker, M. Albiez, M. Taglieber, P. Treutlein, K.-P. Marzlin, and M. K. Oberthaler, Phys. Rev. Lett. 92 (2004) 230401.

[15] H. Sakaguchi, B. A. Malomed, J. Phys. B 37 (2004) 1443.

[16] L. D. Carr, Y. Castin, Phys. Rev. A 66 (2002) 063602; L. D. Carr, J. Brand, Phys. Rev. Lett. 92 (2004) 040401.

[17] D. Mihalache, D. Mazilu, B. A. Malomed, F. Lederer, Phys. Rev. A 73 (2006) 043615). 